\title{
MoCRiS a low-cost stratospheric balloon platform to measure the particle flux of cosmic ray showers in the high atmosphere
}

\section{Valerio Bocci ${ }^{a},{ }^{*}$, Antonino Brosio ${ }^{b}$, Gabriele Galbato Muscio $^{e}$, Francesco lacoangelia , Domenico Liguoric, ${ }$, Maria Antonia Tripodi ${ }^{b}$, on behalf of the OCRA collaboration}

(a complete list of authors can be found at the end of the proceedings)

a INFN sezione di Roma1, P.le Aldo Moro 2, Rome(RM), Italy

b ABproject, Via De Zerbi 3, Rosarno (RC)

c Liceo Scientifico Stefano Patrizi, Via Golia s.n.c., Cariati (CS),Italy

d INFN Gruppo collegato Cosenza, Via P. Bucci Arcavacata di Rende (CS),Italy

e Università di Roma Sapienza, P.le Aldo Moro 2, Rome(RM), Italy

E-mail: valerio.boccieromal.infn.it

\begin{abstract}
In June 2019 from the region of Calabria (Italy) our group launched the Stratospheric Balloon MoCRiS (Measurement of Cosmic Ray in Stratosphere) that reached up to 35111 meters. MoCRiS is a didactical project that uses a Light Balloon with a payload of only $2.1 \mathrm{Kg}$ payload box included. The capsule was Lithium-ion batteries operated and fully instrumented with two Hi-Res Camera, one for picture and one for video, GPS Tracker, two Particle detectors, and sensors for temperature and pressure. Two ArduSiPM [6] scintillation detectors, made by the INFN group in Rome, were used as cosmic ray flux meters. The technology used for these types of devices makes them very economical, light $(<150 \mathrm{~g})$, and with low power consumption $(<1 \mathrm{Watt})$. These characteristics are optimal for use with the balloon and in space (e.g. in CubeSat), and very attractive for educational use, even on the ground. During the school year, the students of the scientific high school "Stefano Patrizi" of Cariati (CS) Italy, participated in the definition of the project following theoretical and practical lessons on aerospace techniques and particle physics with regard to cosmic ray showers in the atmosphere. The mission was completely successful by experimentally verifying the pressure-to-height relation, the height of the thermal inversion in the atmosphere, reaching and exceeding the maximum Regener-Pfotzer level of the shower development. The instrumental and didactic platform created is easily replicable even with further upgrades. We are expanding the collaboration with other schools for further launches.
\end{abstract}

37th International Cosmic Ray Conference (ICRC 2021)

July 12th-23rd, 2021

Online-Berlin, Germany 


\section{Introduction}

The measurements of cosmic ray flux in the atmosphere began with Physicists Victor Francis Hess between 1911 and 1913[1]. The result of Hess showed the level of radiation considerably increase with altitude; at $5.4 \mathrm{~km}$ altitude being about twice as much as at sea level. As a conclusion of his work, he rightly hypothesizes this radiation is not terrestrial in origin but came from outer space and is attenuated by passing the various layers of the atmosphere. In 1925, Robert Andrews Millikan confirmed the discovery giving this radiation the name of "cosmic rays". Such discovery brought Hess to the Nobel prize in 1936.

In 1930s the physicists Eric Regener, first alone and then with his student Georg Pfotzer [5], performed a series of measurements of physical parameters in high atmosphere, including the cosmic ray flux, thanks to the new technology of unmanned rubber balloon, able to reach 30000 meters. In 1932, with an electrometer and a photographic camera to record the measurements, Regener [2] discover as the ionization increase up to an air pressure of $100 \mathrm{mmHg}(13 \mathrm{kPa})$ and then slow down, noting a trend suggesting an asymptote or a maximum (Fig. 1). In 1934, with the collaboration of Georg Pfotzer, instead of an electrometer they used a Geiger counter as a radiation detector[3] and found data consistent with the measurements made in 1932.

Finally, in 1935, they performed a series of measurements with a new apparatus equipped with three Geiger tubes aligned to look for coincidences. The measurement was carried out up to an atmospheric pressure of about $40 \mathrm{mmHg}(5.3 \mathrm{kPa})$ [4]. They saw for the first time the maximum of cosmic ray flux at $100 \mathrm{mmHg}(13 \mathrm{kPa})$, at an altitude of about 17000 meters, and a subsequent decrease of the counts at a higher altitude (Fig. 2).

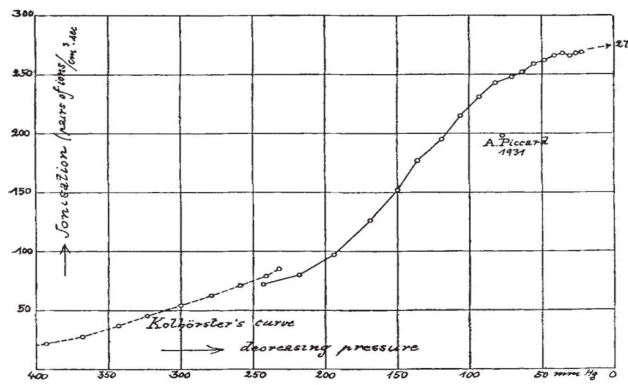

Fig. 1: The first E.Regener measurements published on Nature in 1932[2]. Intensity of cosmic radiation as function of air pressure, measured with an electrometer.

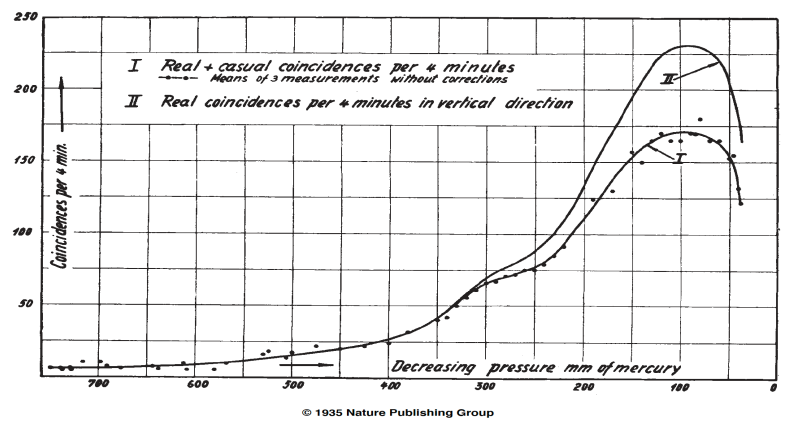

Fig. 2: The number of coincidences per minute as function of air pressure with (II) and without (I) coincidence [4]

The maximum of Regener-Pfotzer is understood; it is due to cosmic ray extensive air showers development. The secondary cascade starts at the upper atmosphere and gradually have a maximum radiation at about $15-20 \mathrm{~km}$ of altitude where the generation of the secondary radiation is balanced by the loss effects; from this point the number of particles starts to decrease.

The idea of the EOS (our first pilot balloons launch done in 2018) and MoCRiS experiments arises from the opportunity to repeat the measurements made by Hess and by Regener and Pfotzer with cheaper but sophisticated equipment, working with high school students. This is possible thanks to the development of sensors, particle detectors, electronic systems, and the availability of Global Navigation Satellite System and light battery. We used, as particle detectors, the ArduSiPM; an all-in- one scintillation detector developed in INFN Rome and innovative in terms of cost, size, weight, and consumption (CSWaP).

\section{Materials and Methods}




\subsection{MoCRiS Ballon parachute and spacecraft}

To be compliant with the less stringent Rules of the Air of International Civil Aviation Organization (ICAO) we chose to use as balloon size a "light" unmanned free balloon which can carries a payload with a combined mass up to $4 \mathrm{~kg}$. A Latex High-Altitude Balloon, with a weight of $1200 \mathrm{~g}$ and filled with about $8 \mathrm{~m}^{3}$ of Helium, was used. The nominal bursting pressure was of $7.3 \mathrm{hPa}$ corresponding to a quote between 29000 meters and 33500 meters. An octagonal spacecraft of $230 \mathrm{~g}$ of polystyrene, 3D printed, with attached a Parachute of $127 \mathrm{~cm}$, held the instrumentation. The ascension speed was about $5 \mathrm{~m} / \mathrm{s}$ and the descendent speed of $20 \mathrm{~m} / \mathrm{s}$. The diameter of the Balloon was 1.8 meter at the beginning and reach $8.6 \mathrm{~m}$ before the burst.

\subsection{The Spacecraft}

The MoCRiS spacecraft was realized in polystyrene to minimize the weight, to guarantee the thermal insulation of the instrumentation, and to cushion the fall. The plant was octagonal with the central part dedicated to flight computers and lithium battery; the eight peripheral compartments were dedicated to the various instruments.

\subsection{Spacecraft Instruments}

There were several instruments inside the spacecraft; some of these are commercial types and others built specifically for the purpose.

\subsubsection{Cameras}

Two commercial GoPro HERO3 Black edition camera:

- One to take pictures with a resolution of 12.0 MPixel, the camera has been set up to take a photo every 4 seconds (we take 1280 photo during the fly).

- One camera for a Full HD Video.

\subsubsection{Two Recovery GPS systems}

- A SPOT GPS tracker using the Glonass satellite network, to get the position during the flight and in case of landing in an area not covered by the GSM network.

- One GPS using GSM message system as backup.

\subsubsection{On board computer and spacecraft}

The onboard computer acquires temperature and pressure from external sensors and time and coordinates from an aviation grade GPS which can acquire the position up to $50000 \mathrm{~m}$.

\subsubsection{The Scintillator detector ArduSiPM}

ArduSiPM is a particle detector based on the scintillation principle developed by our group INFN in Rome. It is the heart of the MoCRiS mission and in real time measures the number of particles that pass through a $5 \times 5 \times 0.5 \mathrm{~cm}^{3}$ plastic scintillator. Its main feature is that of being an all-in-one detector containing the scintillator, the silicon photomultiplier (SiPM), all the control electronics of the SiPM and the data processing part in a module of a few $\mathrm{cm}^{3}$. Its low volume and weight and low power consumption made its use in space applications very attractive. Thanks to a technology transfer from INFN, it is also commercially available to companies that can produce it under license. Its use in schools and in research environments [7][8][9] makes it a quite widespread object in Italy and in some foreign institutes not only for its affordable cost. 
MoCRiS a low-cost stratospheric

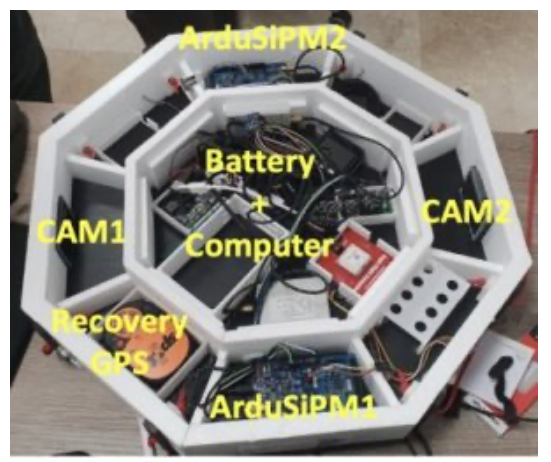

\section{Valerio Bocci}

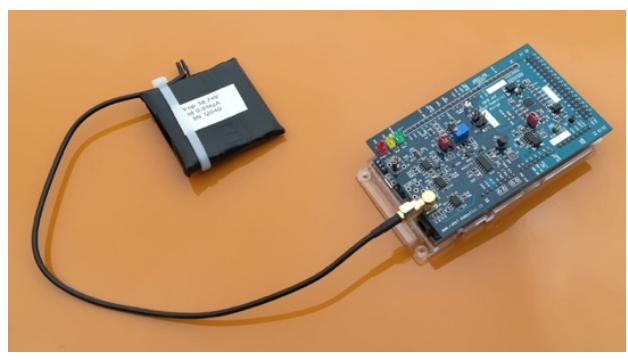

Fig. 4: The ArduSiPM detector

Fig. 3: The octagonal spacecraft with instruments

Two slightly modified ArduSiPM detectors were used for the MoCRiS experiment. A microSD memory was applied to each of the detectors for data $\log$

\subsubsection{The M5Stack module with GPS to setup and acquire ArduSiPM data.}

M5Stack is modular stackable development toolkits based on ESP32 microcontroller with a small 320x240 pixel display. We used an M5 core with attached a GPS M5-GPS and an external pressure and temperature sensor.
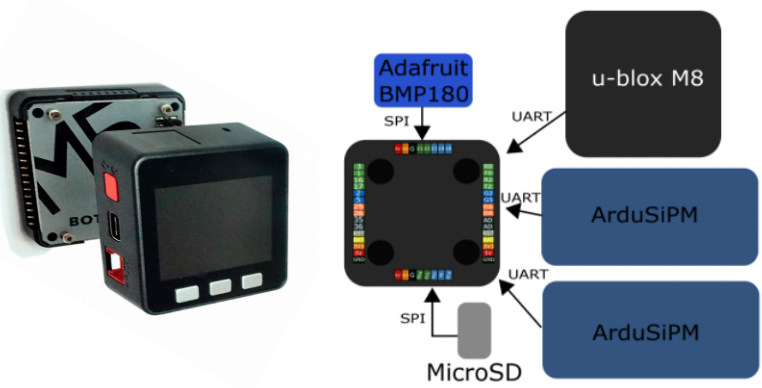

Fig. 5: The M5STACK system for data recording

M5STACK has been programmed to collect the data flows coming from its GPS, from the two ArduSiPMs and from the pressure/temperature sensor and to synchronize them and write data in a third microSD memory. In this way, the system supplies redundancy of data recording units (2 ArduSiPMs, 1 M5Stack, 1 onboard computer).

\section{Launch activities}

The activities of MoCRiS project were carried out with the collaboration of the high school "Liceo Scientifico di Cariati" (CS, Italy). An educational path was developed to teach the basics of cosmic rays physics and to involve the students in the scientific payload construction and in the launch of stratospheric balloon. The launch took place at "località chiesa di San Lorenzo, Spezzano della Sila (CS, Italy)", in close proximity of lake Cecita, on the Sila national park, at 1162 meters of altitude . The place was chosen from some nearby possible areas based on the foreseen path and land place simulated by the software "Predict" (https://predict.habhub.org/). A particular authorization (NOTAM, NOtice To AirMen) was necessary for the balloon activity. Students involved in the project directly participated to launch event, helped the staff to inflate the balloon and carried out the count down in an engaging and immersive event. 
The balloon takes off at 11:24 AM (GMT+2), with a big round of applause, and follow the path recorded by the on-board instrumentation (Fig. 7).

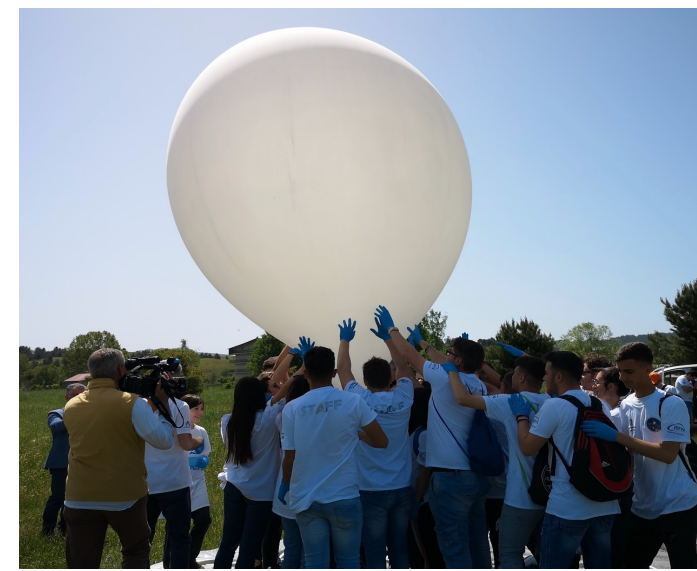

Fig. 6: The launching phase of MoCRiS

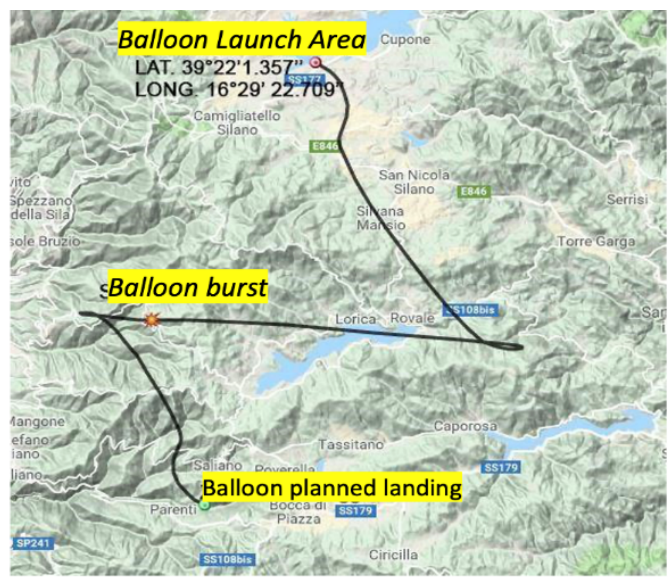

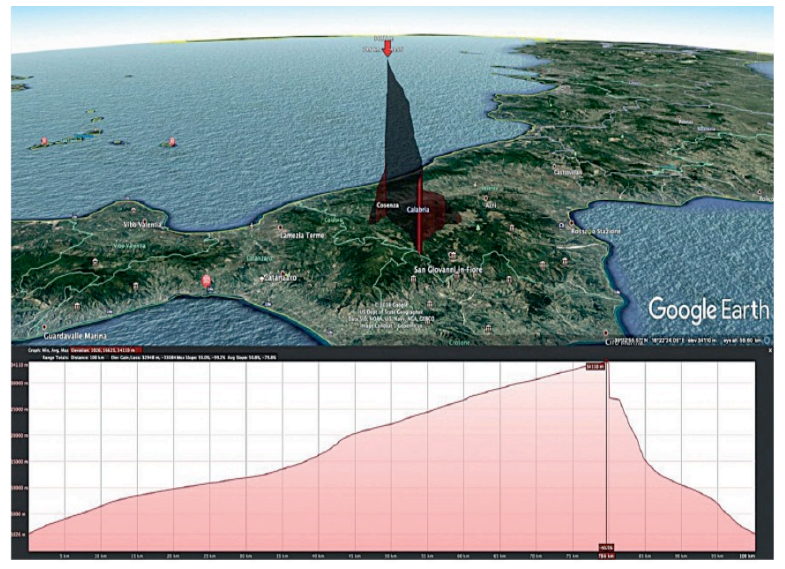

Fig. 7: The balloon path recorded by GPS tracker.

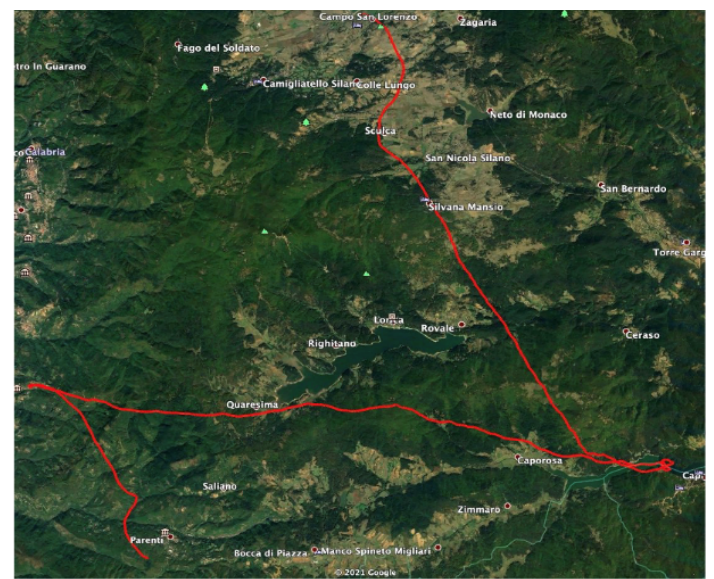

Fig. 8: The balloon path simulated by software (left) and the path recorded by on-board GPS (right)

After 82 minutes it reaches the apogee of its way at the altitude of $34111 \mathrm{~m}$ were the balloon burst in good agreement with the prevision. Next the burst, the scientific payload starts its descend path slowed down thanks to the parachute. The falling trip of scientific payload is faster than the ascent one, it lasts about 23 minutes during which all the instruments continue to record the data. In the end, the payload lands at 01:09 PM, at Parenti (CS, ITALY), in a wooded area, about $25 \mathrm{~km}$ from the take off point: the flight of our probe end.

Unfortunately, the parachute got caught on a about 15 meters high tree and the rescue operation took several hours. Anyway, all the scientific instruments, the ArduSiPMs and all the acquired data were retrieved and shared with the students for analysis.

\section{4. $\quad$ Result}

\subsection{Data analysis}

Once the payload was recovered, all the data recorded from the different instruments was transferred on cloud and put together for offline analysis. 
The presence in MoCRiS of different temperature and pressure sensors made possible the experimental verification of the measurement of these parameters by comparing it with the physics models of the atmosphere.

\subsubsection{Atmosphere temperature}

The air temperature characterizes gaseous planets such as our earth. The dependence is showed on Fig. 9. The temperature slope changing defined the transaction between troposphere and stratosphere.
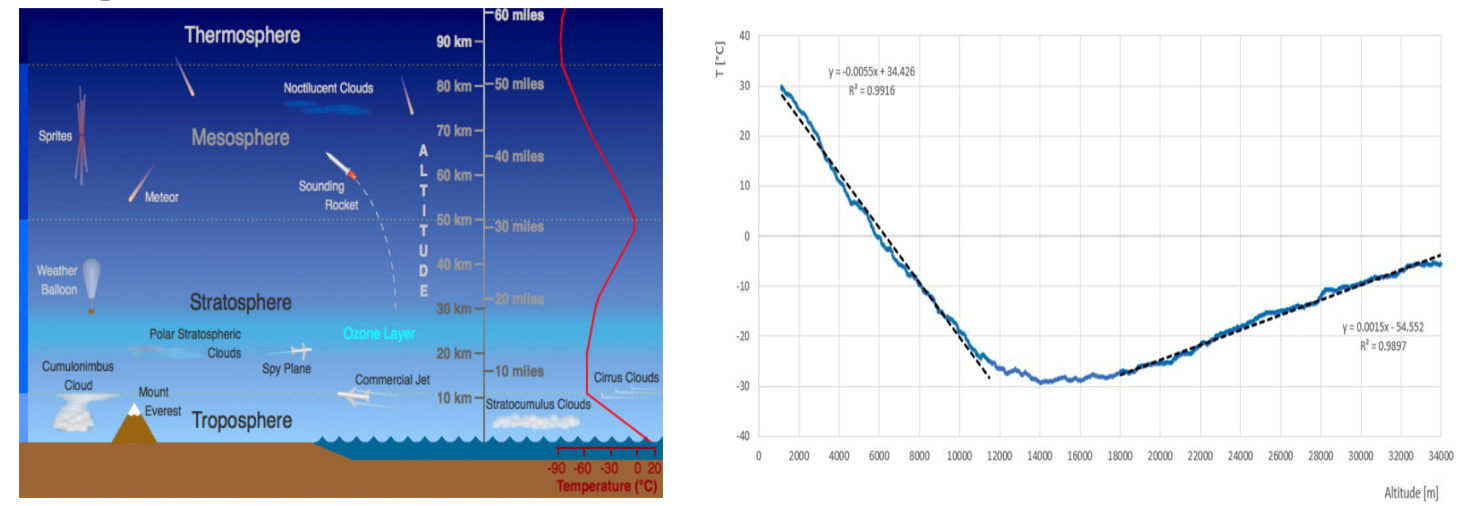

Fig. 9: The Atmosphere layers (Credit:Randy Russell, UCAR) (left) The temperature vs GPS altitude measured from MoCRiS (right)

\subsubsection{Pressure variation with the altitude}

The plot shows the pressure trend as a function of the altitude detected by the GPS system for the ascent phase of the probe. The curves fit well with the simple atmospheric model using the barometric formula. A more fitting parameterization with the experimental data comes out using a three-layer model (Troposphere, Lower Stratosphere, Upper Stratosphere) as suggested by NASA site[13]. Although this model is a simplification of the Earth Global Reference Atmospheric Model (Earth GRAM), it is in perfect agreement with the data collected by MoCRiS.

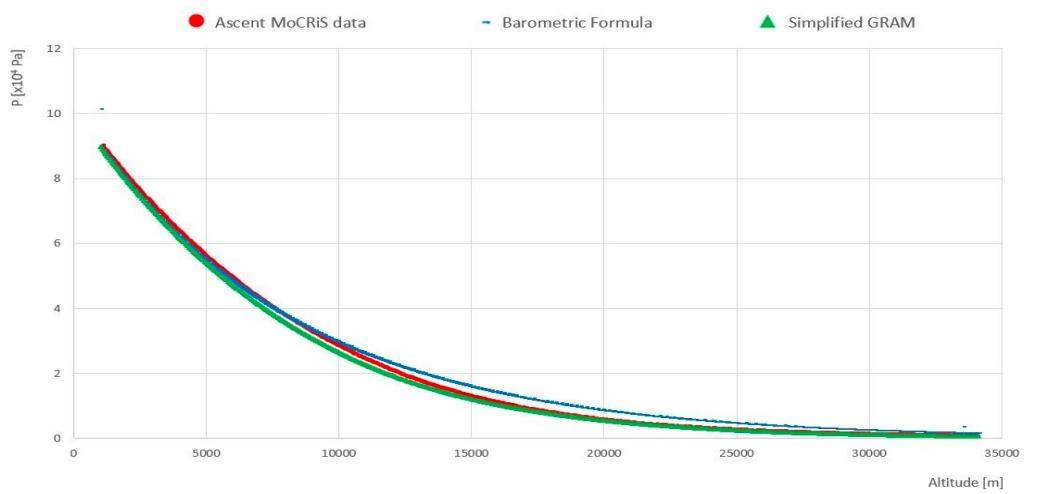

Fig. 10:Pressure dependence from the Altitude measured by MoCRiS compared with different Earth Atmosphere Model

\subsubsection{Ionizing particle flux}

The data about ionizing particles rate from ArduSiPMs system is saved on M5Stack and synchronized with the measures of pression and temperature every one second. The number of ionizing particles detected from each ArduSiPM is plotted as function of atmospheric pressure sensor connected to M5Stack and as function of altitude measured from GPS.

The trend is shape identically to the Regener-Pfotzer measurements of 1935[4]. In particular, the position of inflection point at about $100 \mathrm{mHg}$ is in agreement with the Regener-Pfotzer maximum, to the extent in that it depend of the solar activity. 


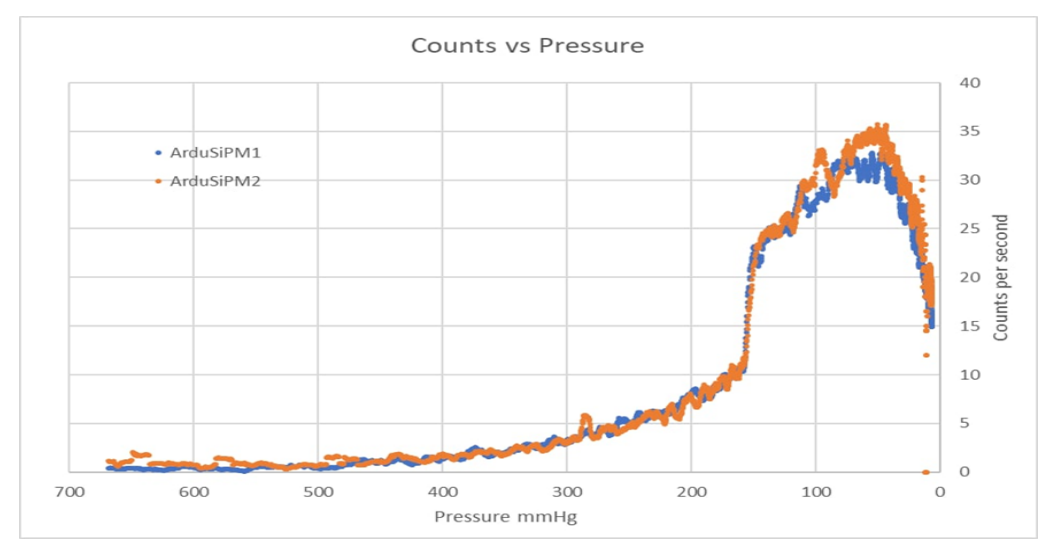

Fig. 11: Plot of ArduSiPMs counts as function of pressure for ascendent path of MoCRiS probe.

\subsubsection{Images from Stratosphere}

As already mentioned, two cameras were part of the MoCRiS instrumentation. Among the thousands of taken photos, this is the most representative and has been chosen by Earth Picture of the Day site (EPOD) as the best picture of June 2019[14]. Taken from a height of $32 \mathrm{~km}$, it shows the waxing crescent Moon above the Italian regions of Calabria, Puglia and Basilicata.

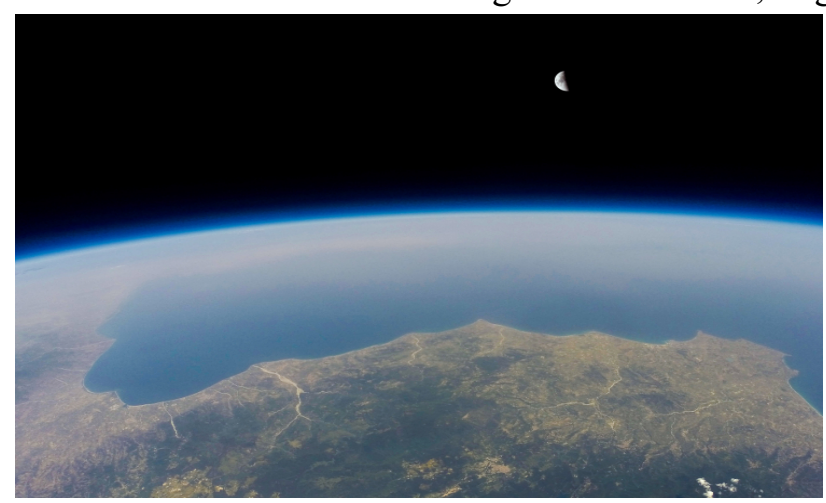

Fig. 12:Mocris picture from $30000 \mathrm{~m}$ granted as best EPOD picture of June 2019

\section{Conclusion}

With the MoCRiS experiment, we have created a low-cost platform for measuring the flux of ionizing particles at high altitudes and validate technologies for use in the upper atmosphere. The platform can be used fruitfully in didactical projects despite the low budget available in schools. The experience acquired with the launch of MoCRiS balloon (and the previous launch of EOS, 2018) has been remarkable. The main critical issues are certainly the choice of the best launch window. In this case, the use of high-altitude current simulators proved to be fundamental and reliable. The return to the land is a critical element but, thanks to the GPS trackers, it was quite easy to find the balloons. Moreover, the finding is only the first step, and the effective recovery can be complicated; as an example, EOS was recovered, one kilometre from the coastline, and MoCRiS on a 15 meters tree. A real-time data downlink would be desirable but in conflict with the cost of satellite uplinks and with strong wireless data transmission regulation. We are considering a wireless data transfer when the probe is visible, after the spacecraft landing and before the real recovery of the hardware components. 
The measurements that can be made aren't limited to radiation but also in other fields, even if not described in the article; in MoCRiS there was a trap for micrometeorites and a small colony of tardigrades that returned unscathed. The communicative impact was considerable and not limited to the class of students who participated. An educational path that uses MoCRiS data is available on the OCRA website[11]. The event was covered by local and national TV, by local newspapers with in-depth articles in national newspapers. Articles in popular science journals[12] have aroused interest with several schools applying for the upcoming launches.

\section{References}

[1] V. F. Hess, Über Beobachtungen der durchdringenden Strahlung bei sieben Freiballonfahrten (English translation A. De Angelis et al) arXiv 1808.02927

[2] Regener, E. Intensity of Cosmic Radiation in the High Atmosphere. Nature 130, 364 (1932). https://doi.org/10.1038/130364a0

[3] Regener, E., Pfotzer, G. Intensity of the Cosmic Ultra-Radiation in the Stratosphere with the TubeCounter. Nature 134, 325 (1934). https://doi.org/10.1038/134325b0

[4] Regener, E., Pfotzer, G. Vertical Intensity of Cosmic Rays by Threefold Coincidences in the Stratosphere. Nature 136, 718-719 (1935). https://doi.org/10.1038/136718a0

[5] Pfotzer, G. History of the use of balloons in scientific experiments. Space Sci Rev 13, 199-242 (1972). https://doi.org/10.1007/BF00175313

[6] V. Bocci, G. Chiodi, F. Iacoangeli, M. Nuccetelli and L. Recchia, "The ArduSiPM a compact trasportable Software/Hardware Data Acquisition system for SiPM detector," 2014 IEEE Nuclear Science Symposium (NSS/MIC), 2014, pp. 1-5, doi: 10.1109/NSSMIC.2014.7431252.

[7] F. Iacoangeli, V. Bocci, G. Cavoto, M. Garattini, L. Recchia, R. Rossi,"Nuclear interaction detector system for UA9 experiments based on ArduSiPM prototype",2015 IEEE Nuclear Science Symposium and Medical Imaging Conference (NSS/MIC), 2015, pp. 1-5, doi: 10.1109/NSSMIC.2015.7581889.

[8] Collamati, F., Bocci, V., Castellucci, P. et al. Radioguided surgery with $\beta$ radiation: a novel application with Ga68. Sci Rep 8, 16171 (2018). https://doi.org/10.1038/s41598-018-34626-x

[9] Calabretta M.M. , Montali L., Lopreside A., Fragapane F., Iacoangeli F., Roda A., Bocci V., D’Elia, $\mathrm{M}$. and Michelini E. Ultrasensitive On-Field Luminescence Detection Using a Low-Cost Silicon Photomultiplier Device Analytical Chemistry 202193 (20), 7388-7393 DOI: 10.1021/acs.analchem.1c00899

[10] G.Galbato Supervisor V.Bocci. Thesis "MoCRiS, studio del flusso di raggi cosmici in stratosfera utilizzando i rilevatori ArduSiPM con pallone libero tipo light"

[11] https://web.infn.it/OCRA/misura-dei-muoni-in-funzione-dellaltezza-in-atmosfera/

[12] Bocci V., Brosio A., Liguori D, MoCRiS: misura di raggi Cosmici in Stratosfera, Astronomia UAIGennaio/Marzo 2020

[13] Earth Atmosphere Model : https://www.grc.nasa.gov/www/k-12/airplane/atmosmet.html

[14] Earth Picture of the Day Moon Imaged from the MoCRiS Payload https://epod.usra.edu/blog/2019/06/moon-imaged-from-the-mocris-payload.html

[15] ArduSiPM site: https://sites.google.com/view/particle-detectors/home 


\section{The OCRA Collaboration}

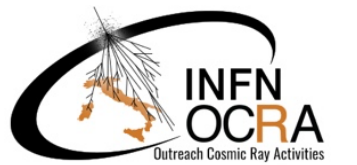

F. Alemanno ${ }^{1,2}$, R. Aloisio ${ }^{1,2}$, C. Altomare ${ }^{3,4}$, R. Antolini ${ }^{1}$, C. Aramo $^{5}$, C. Arcaro $^{6}$, F.C.T. Barbato ${ }^{1,2}$, M. Battaglieri ${ }^{7}$, M. Battisti ${ }^{8,9}$, A. Bau ${ }^{10,11}$, V.E. Bellinzona ${ }^{12}$, P. Bernardini ${ }^{13,14}$, A. Bersani ${ }^{7}$, M. Bertaina ${ }^{8,9}$, A. Berti $^{8}$, B. Bertucci ${ }^{15,16}$, F. Bisconti ${ }^{8,9}$, E. Bissaldi ${ }^{3,4}$, V. Bocci $^{17}$, M. Boezio $^{18,19}$, D. Boncioli ${ }^{1,20}$, M. Bondi $^{7}$, L. Bonechi ${ }^{21}$, R. Bonino ${ }^{8,9}$, G. Bonnoli $^{22}$, V. Bonvicini ${ }^{18}$, E. Bossini $^{22,23}$, B. Bottino ${ }^{7,24}$, M. Buscemi ${ }^{25}$, B. Caccianiga ${ }^{26}$, L. Caccianiga ${ }^{26}$, A. Candela ${ }^{1}$, A. Capone $^{17,27}$, M. Cariello ${ }^{7}$, R. Caruso ${ }^{25,28}$, G. Cataldi ${ }^{13}$, G. Chiodi ${ }^{17}$, G. Chiodini ${ }^{13}$, R. Colalillo ${ }^{5,29}$, M.R. Coluccia ${ }^{13}$, F. Convenga ${ }^{13,14}$, S. Copello ${ }^{7,30}$, M. Corosu ${ }^{7}$, D. D'Urso ${ }^{31,32}$, F. Dal Corso ${ }^{6}$, S. Davini $^{7}$, M. De Deo ${ }^{1}$, S. De Gateano ${ }^{3,4}$, M. De Laurentis ${ }^{5,29}$, I. De Mitri ${ }^{1,2}$, F. De Palma ${ }^{13,14}$, E. De Vito $^{13,14}$, D. Dell'Aquila ${ }^{31,32}$, D. Depaoli ${ }^{8,9}$, A. Di Luca ${ }^{12}$, F. Di Pierro ${ }^{8}$, B. Di Ruzza ${ }^{12}$, M. Di

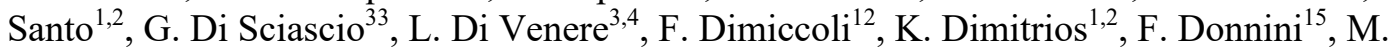

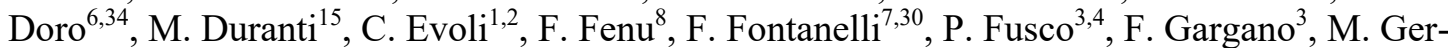

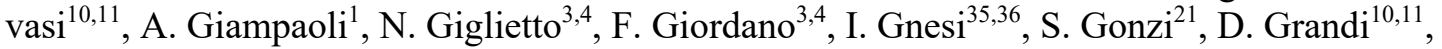
M. Graziani ${ }^{15,16}$, S. Hemmer ${ }^{6}$, F. Iacoangeli ${ }^{17}$, A. Insolia ${ }^{25,28}$, S. Iovenitti ${ }^{26,37}$, V. Ippolito ${ }^{17}$, G. La Vacca $^{10,11}$, G. La Verde ${ }^{5,29}$, E. Leonora ${ }^{25}$, S. Levorato ${ }^{18}$, D. Liguori ${ }^{35,38}$, P. Lipari ${ }^{17}$, F. Longo ${ }^{18,39}$, F. Loparco ${ }^{3,4}$, R. Lopez Coto ${ }^{6}$, S. Loporchio ${ }^{3}$, A. Marino ${ }^{5,40}$, G. Marsella ${ }^{25,41}$, D. Martello ${ }^{13,14}$, M.N. Mazziotta ${ }^{3}$, A. Menegolli ${ }^{42,43}$, S. Miozzi ${ }^{33}$, H. Miyamoto ${ }^{8}$, E. Mocchiutti ${ }^{18}$, S. Morganti ${ }^{17}$, F. Morsani ${ }^{22}$, R. Munini ${ }^{18,19}$, R. Mussa ${ }^{8}$, F. Nozzoli ${ }^{12}$, A. Nucita ${ }^{13,14}$, G.C. Organtini ${ }^{17,27}$, G. Ottonello $^{7}$, F. Pantaleo ${ }^{3,4}$, R. Paoletti ${ }^{22}$, F. Parodi ${ }^{7}$, L. Perrone ${ }^{13,14}$, L. Pesenti ${ }^{10,11}$, S. Petrera ${ }^{1,2}$, C. Petronio $^{35,44}$, R. Pillera ${ }^{3,4}$, F. Pilo ${ }^{22}$, C. Pizzolotto ${ }^{18}$, E. Prandini ${ }^{6,34}$, M. Pugliese ${ }^{5,29}$, S. Rainò ${ }^{3,4}$, N. Randazzo $^{25}$, R. Rando ${ }^{6,34,45}$, L. Recchia ${ }^{17}$, E. Ricci ${ }^{12}$, M. Rinaudo ${ }^{8,9}$, V. Rizi ${ }^{1,20}$, N. Rossi ${ }^{1}$, D. Rozza $^{31,32}$, F. Salamida ${ }^{1,20}$, P. Savina ${ }^{13,14}$, V. Scherini ${ }^{13.14}$, M. Schioppa ${ }^{35,46}$, V. Scotti ${ }^{5,29}$, D. Serini $^{3}$, V. Sipala ${ }^{31,32}$, A. Surdo ${ }^{13,14}$, A. Tiberio ${ }^{21}$, N. Tomassetti ${ }^{15,16}$, C. Tomei ${ }^{17}$, I. Tosta E

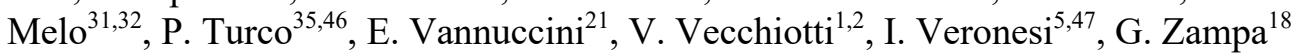

INFN Laboratori Nazionali del Gran Sasso, Assergi, Italy

Gran Sasso Science Institute, L'Aquila, Italy

INFN Sezione di Bari, Bari, Italy

Dipartimento Interateneo di Fisica "M. Merlin", University and Politecnico of Bari, Bari, Italy

INFN Sezione di Napoli, Naples, Italy

INFN Sezione di Padova, Padua, Italy

INFN Sezione di Genova, Genua, Italy

INFN Sezione di Torino, Turin, Italy

Dipartimento di Fisica, University of Turin, Turin, Italy

INFN Sezione di Milano Bicocca, Milan, Italy

Dipartimento di Fisica "Giuseppe Occhialini", University of Milan-Bicocca, Milan, Italy

Trento Institute for Fundamental Physics and Applications, Trento, Italy

INFN Sezione di Lecce, Lecce, Italy

Dipartimento di Matematica e Fisica "Ennio de Giorgi”, University of Salento, Lecce, Italy

INFN Sezione di Perugia, Perugia, Italy

16

Dipartimento di Fisica e Geologia, University of Perugia, Perugia, Italy

17

INFN Sezione di Roma I, Rome, Italy 


\section{MoCRiS a low-cost stratospheric}

\section{Valerio Bocci}

19

20

21

Dipartimento di Fisica, University of Pisa, Pisa, Italy

INFN Sezione di Catania, Catania, Italy

Institute for Fundamental Physics of the Universe, Trieste, Italy

INFN Sezione di Firenze, Florence, Italy

INFN Sezione di Pisa, Pisa, Italy

Physics Department, Princeton University, Princeton, USA

INFN Sezione di Milano, Milan, Italy

Dipartimento di Fisica, University La Sapienza, Rome, Italy Dipartimento di Fisica, University of Genua, Genua, Italy INFN Laboratori Nazionali del Sud, Catania, Italy INFN Sezione di Roma Tor Vergata, Roma, Italy

INFN Sezione di Trieste, Trieste, Italy

Dipartimento di Scienze Fisiche e Chimiche, University of L'Aquila, L'Aquila, Italy

Dipartimento di Fisica e Astronomia "E. Majorana”, University of Catania, Catania, Italy Dipartimento di Fisica "Ettore Pancini", University of Naples "Federico II", Naples, Italy Dipartimento di Chimica e Farmacia, University of Sassari, Sassari, Italy Dipartimento di Fisica e Astronomia “G. Galilei”, University of Padua, Padua, Italy INFN Laboratori Nazionali di Frascati, Gruppo Collegato di Cosenza, Cosenza, Italy Centro Ricerche Enrico Fermi, Rome, Italy Dipartimento di Fisica “A. Pontremoli”, University of Milan, Milan, Italy Liceo Patrizi, Cariati, Italy Dipartimento di Fisica, University of Trieste, Trieste, Italy CNR - Istituto di Scienze Applicate e Sistemi Intelligenti “Eduardo Caianiello”, Pozzuoli, Italy Dipartimento di Fisica e Chimica "Emilio Segrè", University of Palermo, Palermo, Italy INFN Sezione di Pavia, Pavia, Italy Dipartimento di Fisica, University of Pavia, Pavia, Italy Liceo Volta, Reggio Calabria, Italy Centro di Ateneo di Studi e Attività Spaziali “G. Colombo”, University of Padua, Padua, Italy Dipartimento di Fisica, University of Calabria, Arcavacata di Rende, Italy Dipartimento di Matematica, University of Salerno, Salerno, Italy 